\section{Silberschutz für Ekzempatienten}

B ei Patienten mit atopischer Dermatitis können Spezialtextilien mit integrierten Silberfasern Superinfektionen verhindern und somit Juckreiz reduzieren. Die Fasern setzen geringe Mengen an Silberionen frei, die schwefelhaltige Proteine irreversibel binden und so antibakteriell wirken. Wird das Silber zu schnell ausgewaschen, verliert die Textilie ihre keimtötenden Eigenschaften. Andererseits kann sich ein hoher Silberanteil nachteilig auf den Tragekomfort auswirken. Die Metallfasern kratzen oder sie lösen sich von den Textilen und verursachen selbst Juckreiz.

Diese Probleme vermeidet weitgehend die Funktionswäsche der Marke Sansita ${ }^{\circledR}$. Im Gegensatz zu anderen silberhaltigen Textilien reicht bei dieser Wäsche mit eingearbeiteten smartcel ${ }^{\mathrm{TM}}$ -
bioactive-Funktionsfasern ein geringer Silberanteil von nur $2 \%$ aus, um eine effektive antibakterielle Wirkung zu entfalten, die auch nach über 200 Waschgängen noch anhält. Tests des Forschungsinstitut Hohenstein ergaben, dass innerhalb von 18 Stunden über 99\% zuvor aufgetragener Bakterienstämme abgetötet werden. Zusätzlich zur Verringerung des Silberanteils wird dem Tragekomfort durch den Einsatz von besonders weichen und leichten Naturprodukten Rechnung getragen, die frei von Schadstoffen und nach den Qualitätsanforderungen des Öko-Tex Standard $100 \mathrm{ab}$ dem frühesten Kindesalter geeignet sind.

Das Fachklinikum Borkum und die Allergie- und Asthma- Klinik in Bad Lippspringe führten unabhängig voneinander Anwendungsbeobachtungen mit Sansita ${ }^{\circledR}$-Funktionswäsche bei Patienten mit atopischer Dermatitis durch. In beiden Untersuchungen berichteten die Patienten von einem angenehmen Tragegefühl, einer Minderung des Juckreizes und einer deutlichen Verbesserung der Schlafqualität. In Borkum konnten im Verlauf von zwölf Wochen alle Patienten ihren Bedarf an symptomatischer Therapie mit topischen Glukokortikoiden und oralen Antihistaminika reduzieren. Durch Kratzen entzündlich veränderte Hautintegumente bildeten sich vielfach zurück.

Danach kann das regelmäßige Tragen der Spezialtextilien einigen Betroffenen den Alltag erleichtern und zu einer Verbesserung des Hautbilds führen. Weitere Informationen sind im Internet unter www.sansita.de zu finden.

Nach Informationen von Sansita ${ }^{\circledR}$, Haigerloch-Owingen

\title{
Mehr Luft dank MONICA
}

E ine adäquate Asthmakontrolle ist bei vielen Patienten trotz der Therapie mit einem inhalativen Glukokortikoid oder sogar der kombinierten Gabe von Glukokortikoid und lang wirksamen $\beta$ Agonisten nicht möglich. In den jetzt publizierten Ergebnissen der Studie „Montelukast in chronic asthma“, kurz MONICA, konnte gezeigt werden, dass sich durch die zusätzliche Behandlung mit dem Leukotrienantagonisten Montelukast $\left(\right.$ Singulair $\left.{ }^{\circledR}\right)$ patientenrelevante Parameter verbessern lassen (Virchow JC et al. Respir Med 2009 Dec 23 [Epub ahead of print]).

An MONICA waren 1.681 erwachsene Patienten mit leichtem bis mittelschwerem Asthma beteiligt, deren Erkrankung unter der bisherigen Medikation - inhalatives Glukokortikoid allein oder in Kombination mit einem lang wirksamen $\beta$-Agonisten - gemäß der GINA-Leitlinie nicht ausreichend kontrolliert war. Sie erhielten zusätzlich einmal täglich Montelukast $10 \mathrm{mg}$ über einen Zeitraum von sechs Monaten. Wichtigstes Messinstrument war der in der GINA-Leitlinie empfohlene Asthma
Control Test (ACT ${ }^{\mathrm{TM}}$ ), bei dem mittels fünf patientengerechter Fragen der Grad der Asthmakontrolle überprüft wird. Der erzielbare Score variiert zwischen den Werten 5 (Asthma völlig unkontrolliert) und maximal 25 (komplette Kontrolle).

Im Verlauf der Studie wurden signifikante Verbesserungen sowohl im

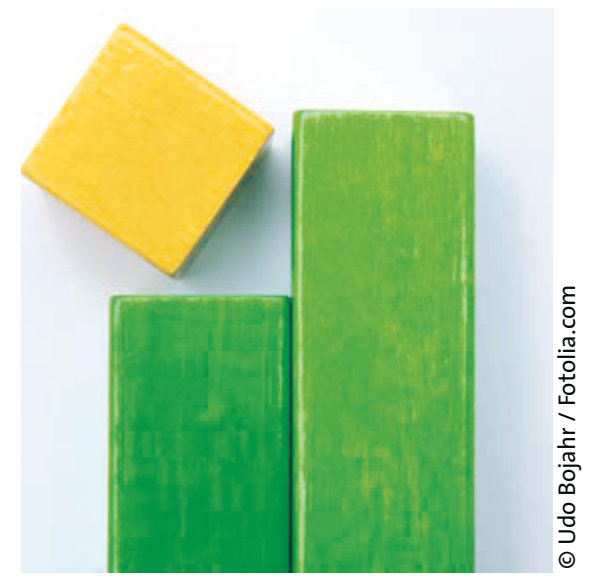

Immer mehr Daten deuten darauf hin, dass die Therapie mit Leukotrienantagonisten ein kleiner, aber wichtiger Baustein zum Erreichen einer guten Asthmakontrolle ist.
Gesamtscore als auch bei allen fünf Einzelfragen erzielt. Während vor der Einnahme von Montelukast nur $15 \%$ der Studienteilnehmer einen ACT-Summenscore von 20 oder mehr aufwiesen, waren es nach sechs Monaten unter der zusätzlichen Behandlung mit Montelukast $59 \%$. Die Lebensqualität, die mit dem „Mini-Asthma Quality of Life Questionaire" erfasst wurde, verbesserte sich parallel dazu in allen Bereichen signifikant, von der Symptomatik über die körperliche Aktivität bis hin zum emotionalen Befinden: Der Gesamtscore stieg von im Mittel 4,0 auf 5,3 $(p<0,0001)$. Auch die Lungenfunktion, gemessen als Einsekundenkapazität FEV1 oder als Peak-Flow-Wert, besserte sich unter Zusatzbehandlung mit Montelukast signifikant ( $<<0,0001)$. Bemerkenswert war außerdem, dass übereinstimmend $83 \%$ der Patienten und 83\% der behandelnden Pneumologen den Asthmastatus nach sechs Monaten zusätzlicher Montelukast-Therapie als „besser“ oder „viel besser“ beurteilten. Eine deutliche Verbesserung war bereits nach den ersten drei Monaten feststellbar.

Nach Informationen von MSD, Haar 